\title{
STUDY OF MOLECULAR DYNAMICS OF CONDENSED STATES OF A SUBSTANCE BY SPECTROSCOPY
}

\begin{abstract}
This work is a logical continuation of our studies [1,2] and devoted to studying the dependence of molecular interactions of toluene and aniline within the wide range of frequencies. As a result of the statistical treatment of the far parts of the depolarized component spectrum of molecular scattering, it has been shown that the best approximation corresponds to a Gaussian.

Keywords: orientation spectroscopy, molecular scattering of light, orientational motion of molecules in liquids, anisotropic molecules, relaxation mechanism, relaxation time, fluctuation.
\end{abstract}

\section{Introduction}

Oscillation orientation spectroscopy is one of the more informative experimental methods of studying the liquid state of a substance and gives information that cannot be frequently obtained by other methods.

The study of the structure of the line wing depolarized component of molecular scattering of light (LWDCMSL) allows one to obtain information about the orientational motion of molecules in liquids.

The fact that LWDCMSL in liquids is very complicated is a strictly established fact. It was experimentally proved that the central contour part (0$50 \mathrm{~cm}^{-1}$ ) has a dispersion (Lorentz) character. Moreover, the large length of LWDCMSL in liquids and solutions (up to $250 \mathrm{~cm}^{-1}$ ) was established. The dispersion contour cannot explain such a length, because this contour has no second moment. Hence, in the real physical processes, there should be a relaxation mechanism cutting the wing.

There are many experimental studies of the LWDCMSL spectral composition. If the central part of the LWDCMSL contour has the dispersion shape for the liquids composed of anisotropic molecules, then there is no such unambiguity for the far parts of LWDCMSL. Since the literature data on the far parts of LWDCMSL are very conflicting [3,4], we scrutinized the intensity distributions of the far parts of LWDCMSL (50-200 $\mathrm{cm}^{-1}$ ) in some organic liquids.

\section{Experimental}

The spectra of the depolarized component of molecular scattering of light were registered with an exper-

(C) SH. OTAJONOV, B. ESHCHANOV,

A. ISAMATOV, 2014

254 imental setup (Figure) assembled on the basis of a spectrometer DFS-12. The setup is intended for measuring the weak glow spectra in an automatic mode (the detailed description of the setup is given in [5]. A He-Ne laser (628-nm radiation wave length) with a power of about $50 \mathrm{~mW}$ was used as a source of radiation. In the spectrum registration system, LWDCMSL is incident on a cooled photocathode FEU-79 operating in the mode of photon counting. To select a working voltage of the photocathode, the counting characteristic curve was measured for various voltages. It was found that the plateau of the counting characteristic curve is within the voltage range 1800$1850 \mathrm{~V}$. For FEU voltage $1800 \mathrm{~V}$, the dark count was 52.2 pulses for a second at room temperature.

A special attention should be paid to the substance purity for LWDCMSL to be studied. In connection with that, there is a serious problem of how to purify a scattering medium from contaminating impurities to have an optically "pure" medium. Therefore, the substances we used were cleaned, dried, dust-removed, and degassed by double vacuum distillation to a dish.

\section{Results and Discussion}

The objects for study were mainly selected, on the one hand, by a likeness of their structure (a benzene ring as a base) and, on the other one, by a difference in molecule shapes and substitution characters. The different character of the attachment of methyl and haloid group to the benzene ring makes them interesting for the study of the effect of a substitute on the character of the relaxation processes of molecules in the liquid phase. Therefore, we selected the liquids composed of the molecules similar in symmetry and

ISSN 2071-0194. Ukr. J. Phys. 2014. Vol. 59, No. 3 


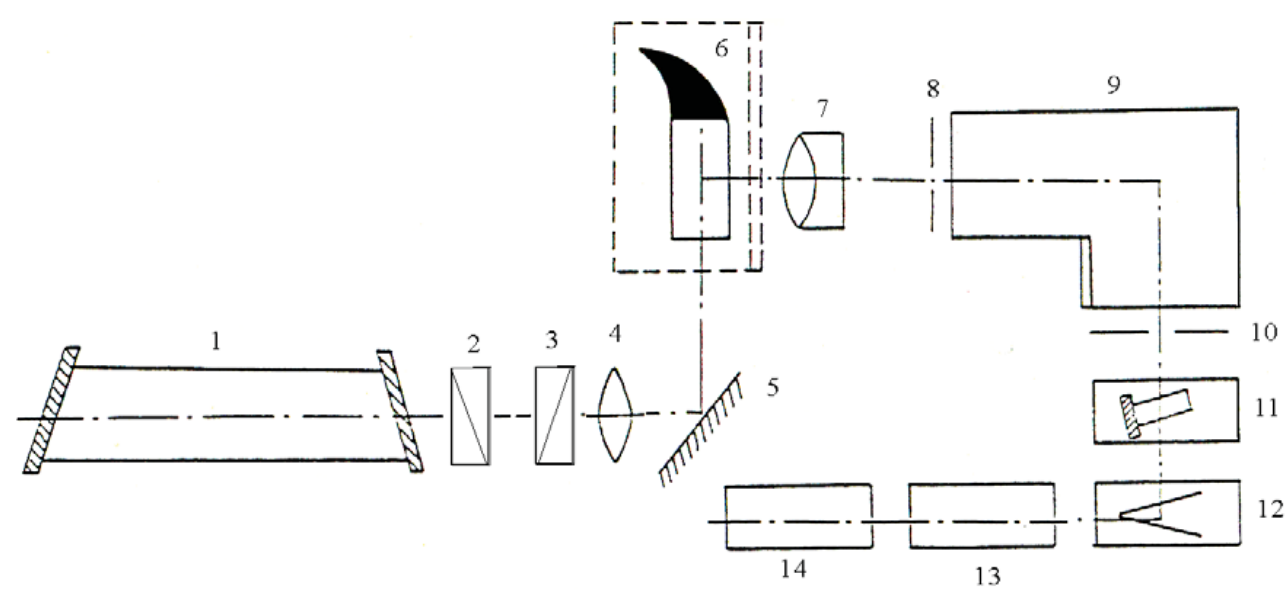

Scheme of the experimental setup for photoelectric registration of the spectra of the depolarized component of molecular scattering of light. 1 - He-Ne laser LG-38; 2, 3-polarizers; 4 - focusing condensator; 5 - rotation mirror; 6 - dish with liquid under study; 7 - condenser; 8 - spectrometer entrance slit; 9 - spectrometer DFS-12; 10 - spectrometer exit slit; 11 -FEU; 12 - emitter repeater; 13 - scaling unit; 14 - digital printer

structure but somewhat different by chemical bonds. They are toluene and aniline; their molecules have the same symmetry $C_{2 V}$ and one base (a benzene ring), but differ in bonds. The result of comparison will show the effect of a molecular structure on the scattering process.

Developed by us, a method of resolving LWDCMSL by its components allows us to establish a number of general regularities for liquids under study. The LWDCMSL contour for toluene and aniline (coordinate $1 / \mathfrak{J}$ of $\omega^{2}$, where $\mathfrak{J}$ is the spectral density of the LWDCMSL contour) is a sum of two dispersion ones in the interval from 0 to $50-60 \mathrm{~cm}^{-1}$ with inflection near $20 \mathrm{~cm}^{-1}$ at room temperature. With a raise in the liquid temperature, the inflection border is shifted to low frequencies. The width of the wide component depends weakly on the temperature. There is an anomaly in the temperature dependence of the width of the narrow component of the contour - an increase in width up to some temperature and a decrease near the critical one. The regularities of the central part of the LWDCMSL contour for toluene and aniline were discussed in detail in [2].

As a result of the statistical treatment of the far parts of the LWDCMSL contour by different analytical functions $\mathfrak{J}(\omega)=\mathfrak{J}_{0} \exp \left(-\frac{\Delta \omega}{\sigma}\right)^{P}$ here $P$ is $(P=1,2)$ and $\mathfrak{J}(\omega)=\mathfrak{J}_{0} \exp \left(-\frac{\Delta \omega}{\sigma}\right)^{2 / 3}$, where $\mathfrak{J}$ is the intensity at maximum, $\sigma$ is the contour half- width), it was shown that the best approximation corresponds to a Gaussian.

The results of calculations for toluene and aniline are presented in Table where $\tau$ is the relaxation time, $P$ is the exponent index, $Z^{2}$ is the root-mean-square deviation of the experimental contour from the calculated one $Z^{2}=\sum\left(\mathfrak{J}_{\exp }-\mathfrak{J}_{\text {cal }}\right)^{2}$.

In our case, the root-mean-square deviation $Z^{2}$ shows the approximation quality. As seen from Table, the best approximation $\left(Z^{2}\right.$ has the least value) is for $P=2$, i.e. the damping of the far parts of LWDCMSL takes place as a Gaussian.

For this regularity to be explained, one makes use of the Fisher's idea to consider the decreasing intensity of the far wings as a manifestation of the dynamics of the initial collision less mode of the time evolution of a scattering system [6]. During the relaxation time, the failure in the phase of some molecules participating

\section{Relaxation times and root-mean-square} deviations calculated for LWDCMSL approximated by the exponent in power $P$ for toluene and aniline for various frequencies $\left(T=200{ }^{\circ} \mathrm{C}\right)$

\begin{tabular}{|c|c|c|c||c|c|c|c|}
\hline$\omega, \mathrm{cm}^{-1}$ & $\tau \times 10^{12}, \mathrm{~s}$ & $P$ & $Z^{2}$ & $\omega, \mathrm{cm}^{-1}$ & $\tau \times 10^{12}, \mathrm{~s}$ & $P$ & $Z^{2}$ \\
\hline \multicolumn{6}{|c||}{ Toluene } & \multicolumn{5}{c|}{ Aniline } \\
$60-150$ & 1.80 & 1 & 45 & $60-150$ & 2.50 & 1 & 70 \\
$60-180$ & 1.30 & 2 & 5.5 & $60-180$ & 2.00 & 2 & 8.5 \\
$60-200$ & 2.00 & $2 / 3$ & 90 & $60-200$ & 2.90 & $2 / 3$ & 120 \\
\hline
\end{tabular}


in fluctuation takes place, which leads to a dephasing. The time changes in the phase of some molecules can be described by the Fokker-Planck diffusion equation. The solution of this equation for small times (high frequency) is the Gauss function.

It was shown that when the temperature increases and the liquid approaches its critical state, the transition interval from a Lorentzian to a Gaussian is shifted to high frequencies, which is another confirmation of the hypothesis that the far wings originate from the dephasing.

Thus, the shape of the high-frequency LWDCMSL makes it possible to get information on a fundamental phenomenon of statistical physics - the phase mixing.

1. Sh. Otajonov, B.X. Eshchanov, and A. Isamatov, Ukr. J. Phys. 56, 1178 (2011).

2. Sh. Otajonov, B.X. Eshchanov, and A. Isamatov, J. Chem. Chem. Eng. 7, 483 (2013).
3. B. Hegemann, K. Baker, and J. Jonas, J. Chem. Phys. 80, 570 (1984)

4. M. Perrot, M. Besnard, J. Lascombe, and M. Bouachir, Can. J. Phys. 59, 1481 (1981).

5. B. Eshchanov, Acta NUUZ, 3, 43 (2005).

6. I.Z. Fisher, Sov. Phys. JETP 54, 289 (1981)].

Received 24.09.13

Ш. Отажсонов, Б. Еичанов, А. Ісаматов

ВИВЧЕННЯ МОЛЕКУЛЯРНӦ̈

ДИНАМІКИ КОНДЕНСОВАНОГО СТАНУ РЕЧОВИНИ МЕТОДАМИ СПЕКТРОСКОПІЇ

Р е з ю м е

Дана робота $\mathrm{e}$ логічним продовженням наших досліджень $[1,2]$ і присвячена вивченню залежності молекулярних взаємодій молекул толуолу і аніліну в широкому частотному інтервалі. У результаті статистичної обробки далеких ділянок спектра деполяризованої компоненти молекулярного розсіювання показано, що найкраща апроксимація відповідає гауссіану. 